\title{
Power Quality Improvements through Power Electronic Interfaced Distributed Generation
}

\author{
Lieven Degroote, Bert Renders, Bart Meersman, Tine L. Vandoorn, and Lieven Vandevelde \\ Department of Electrical Energy, Systems and Automation (EESA), Ghent University (UGent) \\ Sint-Pietersnieuwstraat 41, B-9000 Gent, Belgium \\ e-mail: Lieven.Degroote@UGent.be
}

\begin{abstract}
In low-voltage distribution networks a large amount of single-phase nonlinear loads are connected. This leads to the combined presence of power system unbalance and harmonic distortion. The research presented in this paper focusses on these steady-state power quality problems. It uses a harmonic load flow program, implemented in symmetrical components, to investigate the influence of several single-phase inverter control strategies used to connect any kind of primary energy source to the grid. The influence of these single-phase distributed generation units in the three-phase four-wire distribution network is discussed by means of two recently formulated indicators that combine the power system unbalance and the existing harmonics.
\end{abstract}

Index Terms-Harmonics, Unbalance, Distortion, Distributed Generation (DG), Symmetrical Components

\section{INTRODUCTION}

Power quality issues are an important aspect in power system research. Filters can be placed in order to improve these power quality problems [1], [2]. Next to the use of filters, the increasing presence of single-phase power electronic interfaced distributed generation (DG) units [3] can be applied to improve the power quality in the grid.

Power quality phenomena include all possible situations in which the waveform of the supply voltage or load current deviates from the ideal sinusoidal waveform at the fundamental frequency and with an amplitude corresponding with the nominal value. Moreover, for three-phase systems, the waveforms have to be symmetrical. The power quality can be affected by transient disturbances (voltage sags, voltage swells, impulses, etc.) and steady-state disturbances (harmonics, unbalance, flicker, over- and undervoltages). This paper focusses on the combined effect of harmonics and unbalance phenomena.

The primary energy source is connected to the grid by means of an inverter. In literature, several types of control strategies have been developed for this inverter. This paper will deal with three types of control strategies, namely the ideal sinusoidal control strategy, the real-life sinusoidal control strategy and the damping control strategy. The influence of these types of inverter control strategies will be investigated by means of simulations. The load flow program used for these

The research was carried out in the frame of the Inter-university Attraction Poles programme IAP-VI-021, funded by the Belgian Government. This work is financially supported by the FWO-Vlaanderen (Research Foundation Flanders, Belgium). T. Vandoorn thanks the FWO for the Fellowship received. simulations is developed by the authors in order to simulate any radial low-voltage network. It uses symmetrical components in the harmonic domain and most prevalent devices in the grid are simulated in detail [4]. Next to the use of detailed equivalent circuits, the contribution of this model lies in the use of symmetrical components. Solving the network in symmetrical or in phase components both require solving three networks. However, when phase components are used, the three networks are coupled and consequently, they take the same amount of time to simulate. When symmetrical components are used, the networks are only coupled in the nodes where an unbalanced load is present. Consequently, a change in the positive-sequence voltage component does not result in the same change in currents drawn in the three networks in symmetrical components. This results in a different simulation time for the three networks in symmetrical components, with a shorter simulation time for the zero- and negative-sequence component. So, in the cases with a low degree of unbalance, the overall simulation time is significantly shorter.

An overview of commonly known power quality indices will be described and discussed in the next section. After this, the different inverter control strategies are considered. Finally, in Section IV, the influence of the control strategies in a radial distribution network will be quantified by means of two recently formulated indices.

\section{FramewOrk OF THE POWER Quality Indices}

In literature, several power quality indices have been presented. In what follows, those indices that consider the harmonics and the power system unbalance will be discussed. Most indices have been formulated keeping in mind the negative effect of the deterioration of the power quality. The $X$ mentioned in the following formulas represents the current or the voltage.

Traditionally, distortion has been characterized by the total harmonic distortion indices:

$$
\operatorname{THD}_{\mathrm{X}_{\mathrm{j}}}=\sqrt{\frac{\sum_{h=2}^{\infty} X_{\mathrm{j}, h}^{2}}{X_{\mathrm{j}, 1}^{2}}}
$$

where $X_{\mathrm{j}, h}$ is the rms value of the $h^{\text {th }}$ harmonic order of phase $\mathrm{j}$ (with $\mathrm{j}=\mathrm{a}, \mathrm{b}$ or $\mathrm{c}$ ). An extension of this index is defined 
with the total demand distortion index.

$$
\operatorname{TDD}_{\mathbf{X}_{\mathrm{j}}}=\sqrt{\frac{\sum_{h=2}^{\infty} X_{\mathrm{j}, h}^{2}}{\sum_{h=1}^{\infty} X_{\mathrm{j}, h}^{2}}}
$$

In this way, an index that would be infinite in the case of waveforms without a fundamental component, is avoided [5]. Another way to quantify the harmonic distortion, with the practical relevance of describing the peak current or voltage with respect to the rms value of the signal, is the Crest factor $(\mathrm{CF})$ :

$$
\mathrm{CF}_{\mathrm{j}}=\frac{X_{\mathrm{j}, \text { peak }}}{X_{\mathrm{j}}}
$$

The commonly used index to describe the unbalance is the unbalance factor (UF) which is the ratio of the negativesequence to the positive-sequence:

$$
\% \mathrm{XUF}=\frac{X_{2,1}}{X_{1,1}} \times 100
$$

$X_{1,1}$ and $X_{2,1}$ are the fundamental components of the positiveand negative-sequence voltage or current, respectively. This index gives a straightforward effect of the unbalance on the efficiency of induction machines [6]. Analogously to (4), an unbalance factor can be defined considering the zero-sequence component. This zero-sequence component also diminishes the efficiency of the induction machine as it may give a torque ripple analogously to that of single-phase induction machines.

As a given UF does not result from a unique set of phase voltages or currents, it does not have a unique effect on the efficiency of the machine. Therefore, several subdivisions of the UF have been introduced in [7]. This paper takes the rms value of the positive-sequence component into account. For the same reason, [8] presents the complex unbalance factor (CUF) that considers the phase angle of the different sequence components.

Not every unbalance index uses symmetrical components. For example, the voltage unbalance defined by the National Electrical Manufactures Association (NEMA) is given by:

$$
\frac{\text { Maximum Deviation from Average }}{\text { Average of Three Phase-to-Phase Voltages }} \times 100
$$

In [5], a sinusoidal reference waveform is defined, the rms value of which is the equivalent of the three-phase voltage or current and the deviation index is then given by:

$$
\mathrm{XDI}=\sqrt{\frac{\left|X_{\mathrm{j}}^{2}-X_{e}^{2}\right|}{X_{e}^{2}}} \times 100
$$

where $X_{e}$ is the equivalent rms value of the three-phase waveforms. In [5], three-phase total unbalance indices are defined considering the deviation indices defined in (6). Thereupon, these indices can be weighted and can thus take into account the amount of power transferred by each phase.

Another point of view to quantify unbalance is recently given in [9]-[11]. In these papers, the unbalance factor and the distortion factor are considered together. After all, in almost every practical case, unbalance appears in the presence of harmonic distortion. The indices (5) and (6) also consider the distortion but not in a completely relevant way, namely, the presence of balanced harmonic components could appear as an unbalance. The study of the combined effects of waveform distortion and unbalance in three-phase four-wire systems is aimed at providing useful information for conductor sizing and neutral-point shifting. The indices presented in [10] are formulated in the framework of the symmetrical components. They are based on the calculation of the Fortescue transformation out of the waveforms resulting from the Fourier decomposition. The phase values of the waveforms are given by:

$$
\begin{aligned}
& X_{\mathrm{a}}(t)=X_{\mathrm{a}, 0}+\sqrt{2} \sum_{h=1}^{\infty} X_{\mathrm{a}, h} \cos \left(h \omega t+\phi_{\mathrm{a}, h}\right) \\
& X_{\mathrm{b}}(t)=X_{\mathrm{b}, 0}+\sqrt{2} \sum_{h=1}^{\infty} X_{\mathrm{b}, h} \cos \left(h\left(\omega t+\frac{4 \pi}{3}\right)+\phi_{\mathrm{b}, h}\right) \\
& X_{\mathrm{c}}(t)=X_{\mathrm{c}, 0}+\sqrt{2} \sum_{h=1}^{\infty} X_{\mathrm{c}, h} \cos \left(h\left(\omega t+\frac{2 \pi}{3}\right)+\phi_{\mathrm{c}, h}\right)
\end{aligned}
$$

where $\phi_{\mathrm{j}, h}$ is the phase angle deviation in the reference frame of the considered harmonic. These waveforms (7) are transformed to symmetrical components by using the wellknown symmetrical component transformation matrix [12]:

$$
T=\frac{1}{3}\left[\begin{array}{ccc}
1 & 1 & 1 \\
1 & a & a^{2} \\
1 & a^{2} & a
\end{array}\right]
$$

with $a=e^{j \frac{2 \pi}{3}}$. The complex values of the symmetrical components are calculated for every harmonic order as:

$$
\left[\begin{array}{c}
\underline{X}_{0, h} \\
\underline{X}_{1, h} \\
\underline{X}_{2, h}
\end{array}\right]=T\left[\begin{array}{c}
\underline{X}_{\mathrm{a}, h} \\
\underline{X}_{\mathrm{b}, h} \\
\underline{X}_{\mathrm{c}, h}
\end{array}\right]
$$

The indices discussed below are based on the decomposition of (9) into a balanced and unbalanced part. The balanced part consists of every harmonic sequence component contributing to three waveforms that are periodical (with period $T$ ), equal in shape and have a time shift of $T / 3$ from one phase to the next phase in the a,b,c-sequence. In (7), this means that $X_{\mathrm{j}, h}$ and $\phi_{\mathrm{j}, h}$ are the same for the three phases if the system is balanced. In symmetrical components, this results in the following harmonics that contribute to a balanced set of waveforms, for $h=0,1, \ldots, \infty$ :

- Positive-sequence: The components with harmonic order $3 h+1$

- Negative-sequence: The components with harmonic order $3 h+2$

- Zero-sequence: The components with harmonic order $3 h$ The balanced part $\left(X^{b}\right)$ can be written as:

$$
X^{b}=\sqrt{\sum_{\mathrm{k}=0}^{2} \sum_{h=0}^{\infty} X_{k, 3 h+k}^{2}}
$$


Consequently, the unbalanced part $\left(X^{u}\right)$ is defined as:

$$
X^{u}=\sqrt{\sum_{\mathrm{k}=0}^{2} \sum_{h=0}^{\infty}\left[X_{k, 3 h+(k+1 \bmod 3)}^{2}+X_{k, 3 h+(k+2 \bmod 3)}^{2}\right]}
$$

Next to this balanced and unbalanced part, the waveforms can also be subdivided in a fundamental $\left(X^{1}\right)$ and distorted $\left(X^{d}\right)$ part:

$$
\begin{aligned}
X^{1} & =\sqrt{\sum_{\mathrm{k}=0}^{2} X_{k, 1}^{2}} \\
X^{d} & =\sqrt{\sum_{\mathrm{k}=0}^{2} \sum_{h=2}^{\infty} X_{k, h}^{2}}
\end{aligned}
$$

Furthermore, combinations of these subdivisions can be made. The power quality indices resulting from the above mentioned deduction are the 'Total Phase Distortion':

$$
\mathrm{TPD}_{\mathrm{X}}=\frac{X^{d}}{X^{1}}
$$

and the 'Total Phase Unbalance':

$$
\operatorname{TPU}_{\mathrm{X}}=\frac{X^{u}}{X^{b}}
$$

As described in [11], the TPU indicator provides the correct information also when the classical unbalance indicator is unable to detect unbalance due to the effects of waveform distortion. Therefore, indicators (14) and (15), will be used to analyse the influence of inverter-connected DG units in the low-voltage grid. Moreover, the load flow model used for the simulations has the advantage that the network is already solved in symmetrical components and as such, no transformations are needed to calculate the above-mentioned TPD and TPU.

\section{INVERTER CONTROL STRATEGIES}

In this section, three types of single-phase inverter control strategies, used in this paper, will be described, namely, the damping inverter, an ideal sinusoidal inverter and a real-life or common sinusoidal inverter.

\section{A. The Damping Inverter}

Next to its function as supplier of fundamental active power, the control strategy of the 'damping inverter' has been developed in order to damp harmonic voltage components in the grid voltage [13]. This action is obtained when the inverterconnected DG unit acts as a resistance for the harmonics appearing in the network [14]. This allows the converter to absorb harmonic currents corresponding to the harmonic voltages in the point of connection (POC). The value of these harmonic currents is determined by the programmable damping resistance $1 / g_{\mathrm{h}}$. The conductance $\left(g_{\mathrm{h}}\right)$ is chosen as $P_{\text {nom }} / V_{\text {ref }}^{2}$, with $P_{\text {nom }}$ the nominal power of the inverter and $V_{\text {ref }}$ the reference voltage at the POC. As such, the conductance has a value of $1 \mathrm{pu}$ in the reference frame of the inverter. The

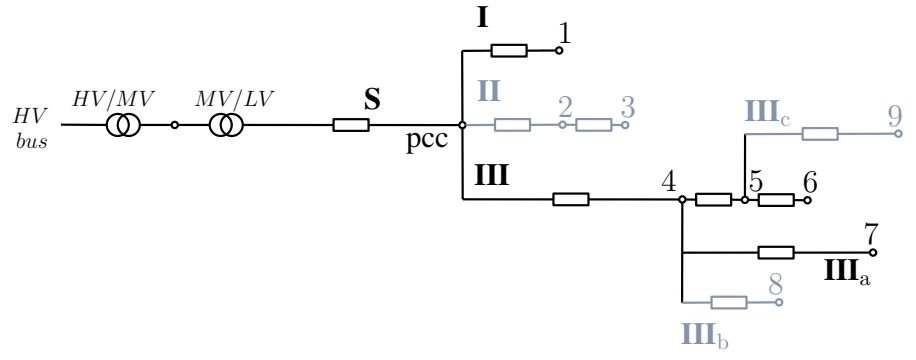

Fig. 1. Topology of the considered radial distribution network.

damping potential of this inverter is thus independent of the active power exchanged with the grid.

In order to simulate this behaviour, the following parameters are required: the nominal power and reference voltage, which determine the damping potential of the inverter $\left(g_{\mathrm{h}}\right)$, the input power from the energy source at the DC-bus of the inverter and the frequency spectrum of the voltage at the point of connection. With these parameters the inverter current can be determined. The amplitude of the fundamental part of the inverter current is calculated so that the fundamental output power of the inverter into the grid equals the power associated with the damping action of the inverter and the power input at the DC-bus coming from the energy source. Herein, the inverter losses are neglected.

\section{B. The Purely Sinusoidal Inverter}

The 'purely sinusoidal inverter' used in the simulations will inject a non-distorted sinusoidal current waveform into the grid, even when the voltage in the POC is distorted. This situation is not realistic as the entrance filter of the inverter will cause harmonic currents to flow in the case of a distorted voltage at the POC. The amplitude of the current is calculated so that the power output of the inverter into the grid equals the power coming from the DC-source.

\section{The Common Sinusoidal Inverter}

In almost every practical case, a non-distorted sinusoidal current waveform will not be achieved as voltage distortion is common in the low-voltage grid. Therefore, the influence of a 'common sinusoidal inverter' will be investigated by using a harmonic fingerprint of a sinusoidal inverter [15]. The harmonic fingerprint of a device describes the interaction between the harmonic voltages and the harmonic currents.

\section{INFLUENCE OF INVERTER CONTROLLED DG UNITS}

The influence of the different single-phase inverter control types will be investigated on the three-phase four-wire network presented in Fig. 1.

The LV network lines are either underground cable lines, encountered mainly in urban areas, or overhead lines. In the network used for the simulations both are present. The cables are represented by grey lines and the overhead lines are represented by black lines (Fig. 1). Every line section consists of four wires (three-phase and neutral) and the properties of the lines are given in Tables I and II. The properties listed 
TABLE I

FEEDER LENGTH AND NOMINAL POWER

\begin{tabular}{|c|cc|}
\hline Feeder & length $(\mathrm{m})$ & $S_{\text {nom }}(\mathrm{kVA})$ \\
\hline S & 620 & 90 \\
I & 155 & 38 \\
II & 248 & 36 \\
III & 806 & 40 \\
III $_{\mathrm{a}}$ & 310 & 9 \\
III $_{\mathrm{b}}$ & 155 & 25 \\
III $_{\mathrm{C}}$ & 248 & 18 \\
\hline
\end{tabular}

TABLE II

THE LINE PROPERTIES

\begin{tabular}{|c|lll|}
\hline Feeder & $\mathrm{R}(\Omega / \mathrm{km})$ & $\mathrm{L}(\mathrm{mH} / \mathrm{km})$ & $\mathrm{C}(\mu \mathrm{F} / \mathrm{km})$ \\
\hline S & 0.0439 & 0.0573 & $/$ \\
I & 0.351 & 0.344 & $/$ \\
II & 0.226 & 0.223 & 0.29 \\
III & 0.0585 & 0.086 & $/$ \\
III $_{\mathrm{a}}$ & 0.351 & 0.344 & $/$ \\
III $_{\mathrm{b}}$ & 0.226 & 0.223 & 0.29 \\
III $_{\mathrm{c}}$ & 0.226 & 0.223 & 0.29 \\
\hline
\end{tabular}

in Table II are valid for the fundamental component. For the higher order harmonics, the resistance has been adjusted according to [16].

Starting from a base case, where no DG units are connected to the grid, several scenarios will be presented in order to study the influence of the different inverter control types on the indices, (14) and (15), presented in Section II. The load distribution for the base case is given in Table III. In this table, the displacement power factor is given (dPF), which describes the power factor using the fundamental frequency components only [17]. The nonlinear loads are simulated based on the research presented in [18].

The state of the grid in the base case is given in Tables IV and $\mathrm{V}$. In these tables, for the voltages, the number on the left is the node, while for the currents, the segment before the node is considered. The influence of the asymmetrical nonlinear load in node 5 can be noticed in these tables. Furthermore, from the different phase values for the $\mathrm{THD}_{\mathrm{I}}$, there can be

TABLE III

THE LOAD DISTRIBUTION

\begin{tabular}{|c|cl|cc|}
\hline & RL-load (kVA) & dPF & nonlinear load (kVA) & phase \\
\hline 1 & 20 & 0.9 & 6 & a-b-c \\
2 & 15 & 0.95 & 5 & a-b-c \\
3 & 7 & 0.9 & $/$ & $/$ \\
4 & 4 & 0.9 & $/$ & $/$ \\
5 & 7 & 0.95 & 3 & a \\
6 & 3 & 0.85 & 1 & a-b-c \\
7 & 2 & 0.9 & 2 & a-b-c \\
8 & 6 & 0.9 & $/$ & $/$ \\
9 & 2 & 0.9 & 3 & a-b-c \\
\hline
\end{tabular}

TABLE IV

THE DISTORTION IN THE BASE CASE

\begin{tabular}{|c|c|ccc|}
\hline & $\mathrm{TPD}_{\mathrm{V}}(\%)$ & $\mathrm{THD}_{\mathrm{V}_{\mathrm{a}}}(\%)$ & $\mathrm{THD}_{\mathrm{V}_{\mathrm{b}}}(\%)$ & $\mathrm{THD}_{\mathrm{V}_{\mathrm{c}}}(\%)$ \\
\hline 1 & 2.40 & 2.54 & 2.32 & 2.32 \\
2 & 1.99 & 2.13 & 1.91 & 1.91 \\
3 & 1.98 & 2.13 & 1.90 & 1.90 \\
4 & 2.55 & 2.90 & 2.36 & 2.37 \\
5 & 2.67 & 3.05 & 2.47 & 2.47 \\
6 & 2.69 & 3.07 & 2.48 & 2.49 \\
\hline & $\mathrm{TPD}_{\mathrm{I}}(\%)$ & $\mathrm{THD}_{\mathrm{I}_{\mathrm{a}}}(\%)$ & $\mathrm{THD}_{\mathrm{I}_{\mathrm{b}}}(\%)$ & $\mathrm{THD}_{\mathrm{I}_{\mathrm{c}}}(\%)$ \\
\hline 1 & 11.82 & 11.78 & 11.83 & 11.84 \\
2 & 9.17 & 9.11 & 9.20 & 9.21 \\
3 & 1.31 & 1.40 & 1.27 & 1.27 \\
4 & 15.86 & 20.68 & 10.20 & 10.20 \\
5 & 22.53 & 28.15 & 12.64 & 12.65 \\
6 & 13.04 & 12.97 & 13.06 & 13.08 \\
\hline
\end{tabular}

TABLE V

THE UNBALANCE IN THE BASE CASE

\begin{tabular}{|c|c|cc|}
\hline & $\mathrm{TPU}_{\mathrm{V}}(\%)$ & $\mathrm{VUF}_{2}(\%)$ & $\mathrm{VUF}_{0}(\%)$ \\
\hline 1 & 0.27 & 0.06 & 0.22 \\
2 & 0.27 & 0.06 & 0.22 \\
3 & 0.27 & 0.06 & 0.22 \\
4 & 0.65 & 0.15 & 0.54 \\
5 & 0.71 & 0.16 & 0.59 \\
6 & 0.71 & 0.16 & 0.59 \\
\hline & $\mathrm{TPU}(\%)$ & $\mathrm{IUF}_{2}(\%)$ & $\mathrm{IUF}_{0}(\%)$ \\
\hline 1 & 0.21 & 0.03 & 0.20 \\
2 & 0.22 & 0.04 & 0.20 \\
3 & 0.25 & 0.06 & 0.23 \\
4 & 14.63 & 9.25 & 9.06 \\
5 & 25.34 & 16.13 & 15.85 \\
6 & 0.53 & 0.07 & 0.50 \\
\hline
\end{tabular}

concluded that the load currents are dependent on the voltage at the point of connection.

\section{A. Point of Connection}

At first, the influence of the three control strategies will be discussed, when the inverter is connected at different locations. Therefore, a single-phase $3 \mathrm{~kW}$ DG unit is connected to phase a in node $1,4,6$ or 8 . In addition, also the combination of these cases has been considered where four single-phase $3 \mathrm{~kW}$ DG units are connected in phase a to the nodes $1,4,6$ and 8 respectively (POC 1, 4, 6 and 8).

In Fig. 2 changes in the total phase voltage distortion $\left(T P D_{V}\right)$ are given with respect to the $T P D_{V}$ in the base case. A negative value for the $\triangle \mathrm{TPD}_{\mathrm{V}}$ corresponds with a decrease of the voltage distortion. On the abscissa the place is given, starting with the source node, followed by the voltage at the secondary of the high voltage transformer, the voltage of the distribution transformer, then the voltage in the pcc and the voltage in the main nodes of Feeder III.

Fig. 2(a) shows the influence of a damping inverter. The following conclusions can be drawn from this figure: 
- The reduction of $\mathrm{TPD}_{\mathrm{V}}$ in the considered nodes for the case where the inverter is connected to node 8 (POC 8 ) is smaller than the case where the inverter is connected to POC 4. This is due to the large impedance between node 4 and node 8 , which causes a harmonic voltage drop, and therefore, it is less efficient to reduce the distortion in node 4. Moreover, in this specific case the higher reduction for POC 1 compared to POC 8 is due to smaller electrical distance to the sources of distortion.

- From POC 4 and POC 6 can be concluded that the effect on the resulting $\triangle \mathrm{TPD}_{\mathrm{V}}$ is better when the inverter is connected more downstream.

- If more inverters are placed along the network (POC 1, 4,6 and 8), then the $\mathrm{TPD}_{\mathrm{V}}$ is more reduced than for the separate cases. This reduction almost equals the relative sums of the separate cases.

When Fig. 2(b) is compared with Fig. 2(a), the influence of the damping effect becomes quite clear. Less reduction is obtained with the purely sinusoidal inverter and it can be totally allocated to the fundamental voltage rise caused by the DG unit. Furthermore, it can be stated that most reduction, in the considered nodes, is obtained when the DG unit is connected to a node or a subfeeder connected to a node more downstream and as such, the reduction for POC 8 is larger than POC 1.

At last, Fig. 2(c), shows the influence of a common sinusoidal inverter. It is shown that a prediction of the $\triangle \mathrm{TPD}_{\mathrm{V}}$ is impossible. This, due to the dependency of the current to the voltage via the harmonic fingerprint. As such, it is possible that the distortion gets worse when the inverter is connected to node 6 , where an improvement is obtained when the inverter is connected to node 4 . So, the conclusions drawn before, do not count for a common sinusoidal inverter. When the distortion of the three-phase voltages for POC 6 is compared with the base case, there can be seen that in phase a, where the DG unit is connected, the $\mathrm{THD}_{\mathrm{V}}$ improves but in the two other phases the $\mathrm{THD}_{\mathrm{V}}$ deteriorates. This is caused by the enlarged harmonic neutral current, resulting in a more distorted phase voltage for phase $\mathrm{B}$ and $\mathrm{C}$. This endorses the need for a single value for the distortion in the grid, as in a first consideration only the distortion in phase a would be considered $\left(\mathrm{THD}_{\mathrm{V}_{\mathrm{a}}}\right)$, resulting in a wrong conclusion about the distortion for the three-phase voltages. Furthermore, from Fig. 2(c) can be concluded that the resulting $\mathrm{TPD}_{\mathrm{V}}$ for several DG units (POC 1, 4, 6 and 8) can not be found by considering the separate cases. The reason lies in a combination of the attenuation and diversity effect occurring between the different harmonic sources [19]. So, when harmonic simulations are performed, phase angles have to be taken into account, otherwise these effects would be neglected.

In Fig. 3 changes in the total phase voltage unbalance $\left(\mathrm{TPU}_{\mathrm{V}}\right)$ are given with respect to the $\mathrm{TPU} \mathrm{V}_{\mathrm{V}}$ in the base case. When comparing the three different control types, one can see that the difference is quite small. This is logical as, if only the traditional voltage unbalance factors would be compared, i.e. when only the fundamental components would be considered,

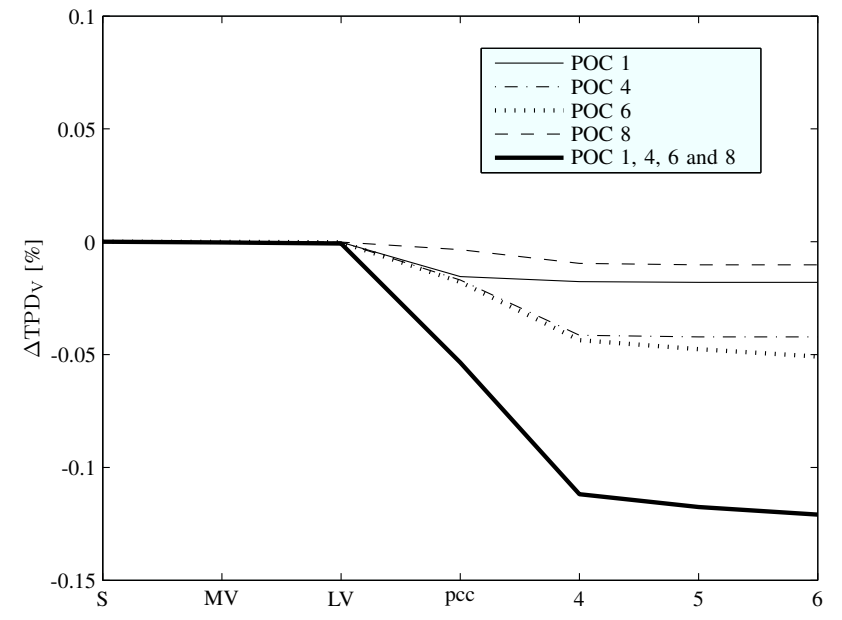

(a) Damping inverter

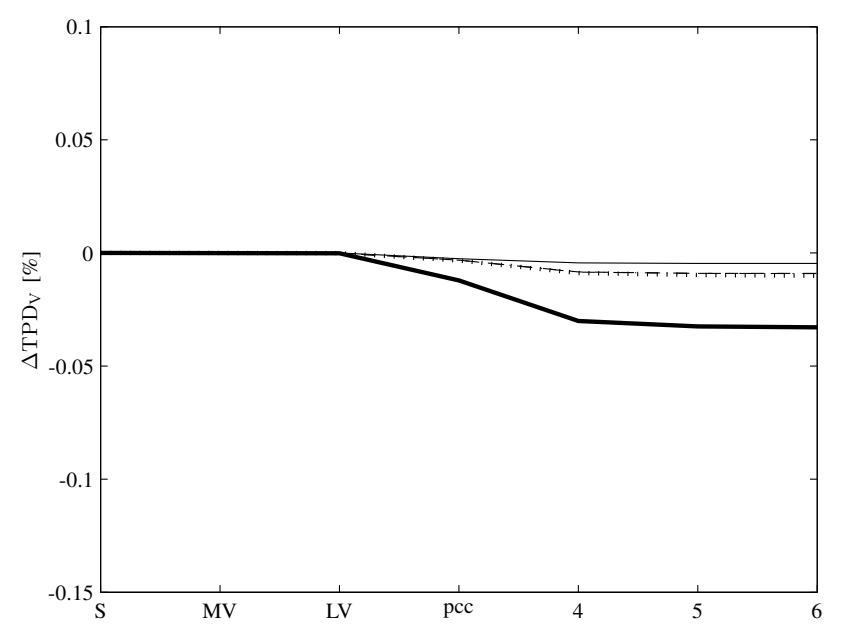

(b) Purely sinusoidal inverter

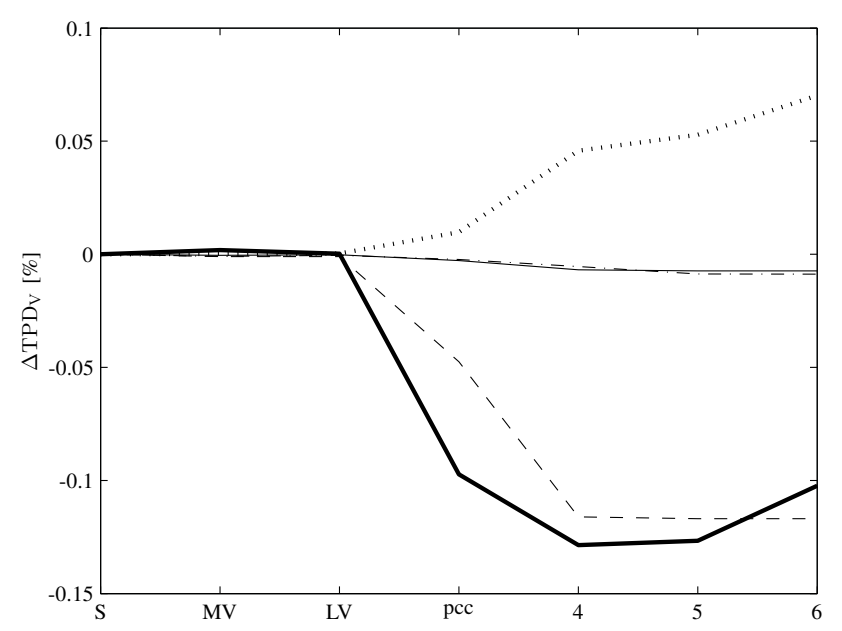

(c) Common sinusoidal inverter

Fig. 2. Influence of the point of connection on the $\triangle \mathrm{TPD}_{\mathrm{V}}$ for different types of inverter control 


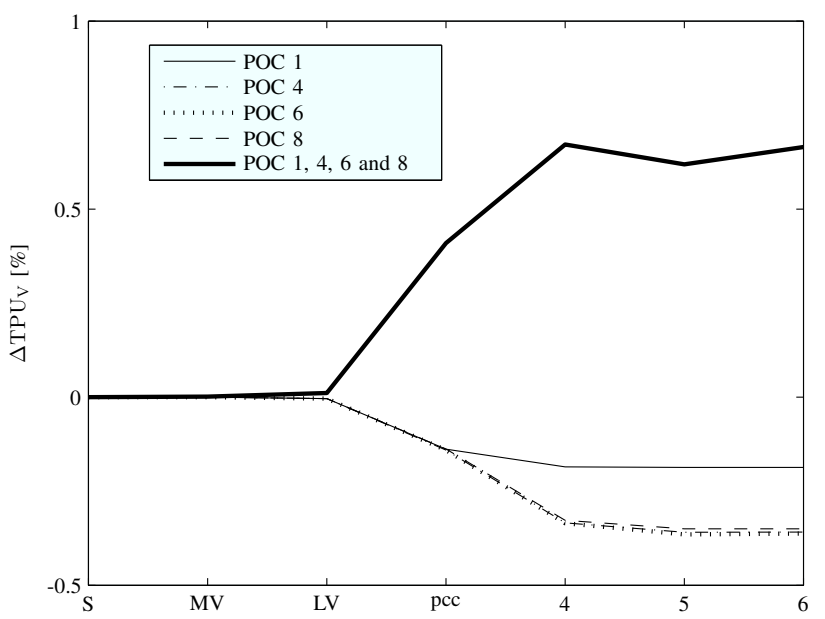

(a) Damping inverter

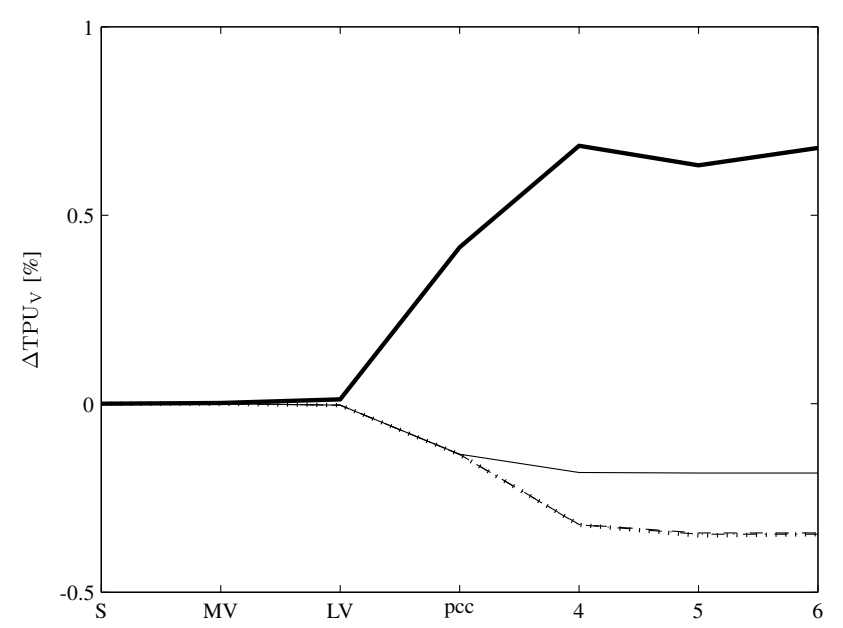

(b) Purely sinusoidal inverter

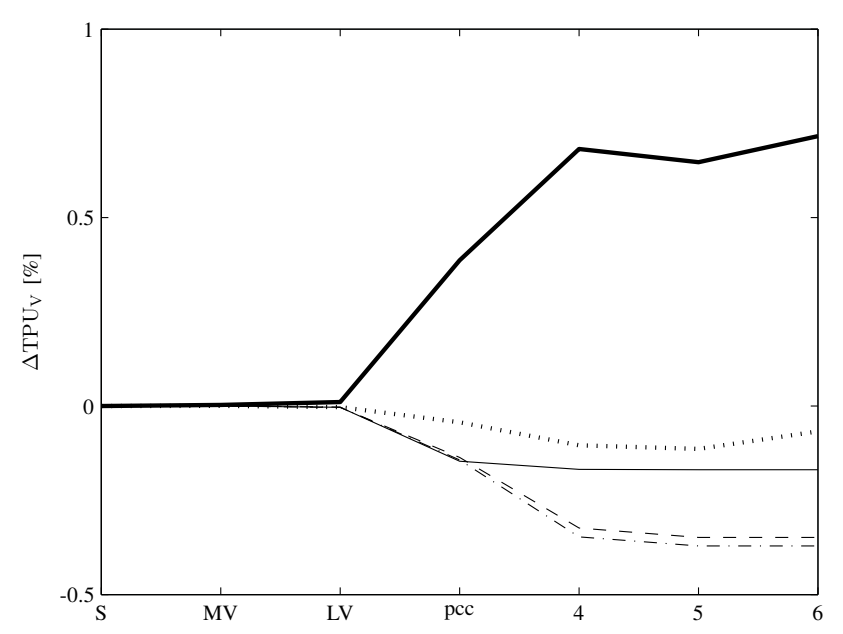

(c) Common sinusoidal inverter

Fig. 3. Influence of the point of connection on the $\triangle T P U_{V}$ for different types of inverter control

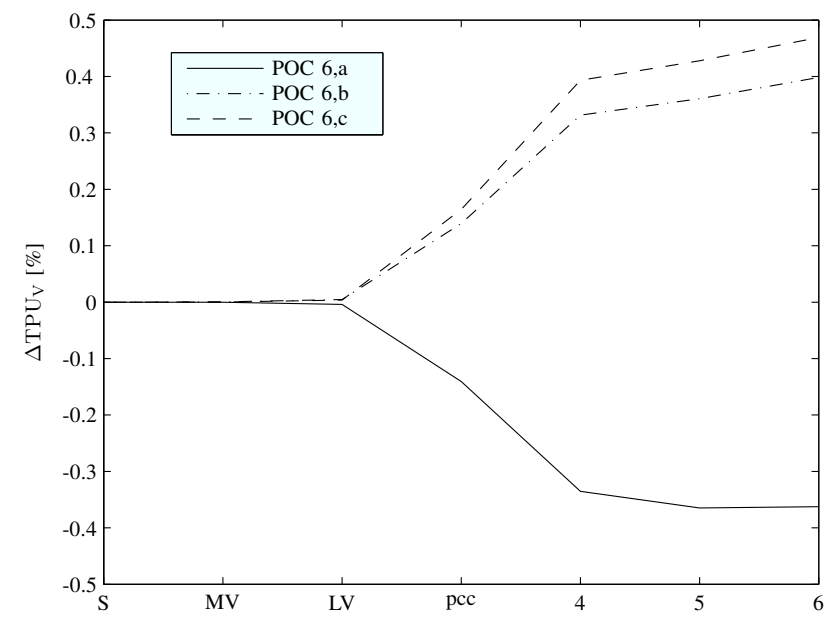

(a) Damping inverter

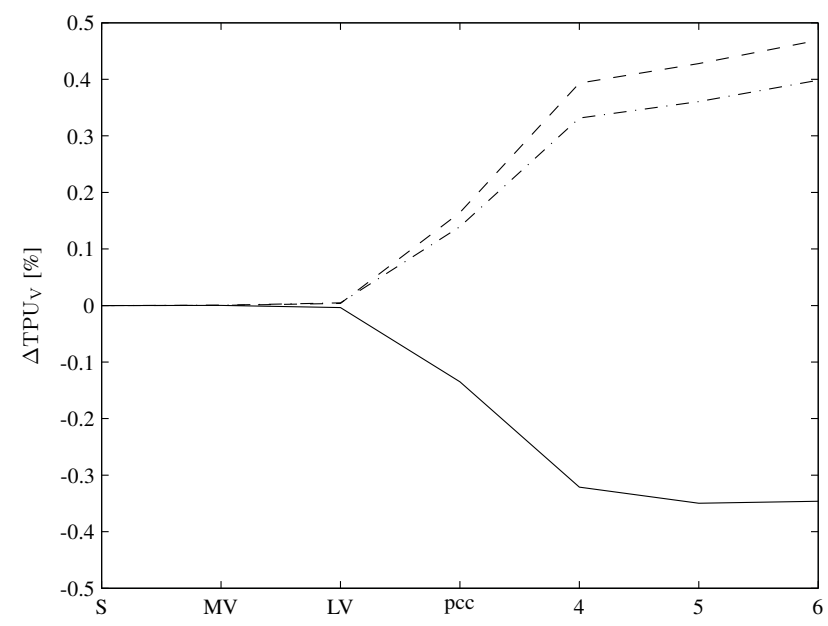

(b) Purely sinusoidal inverter

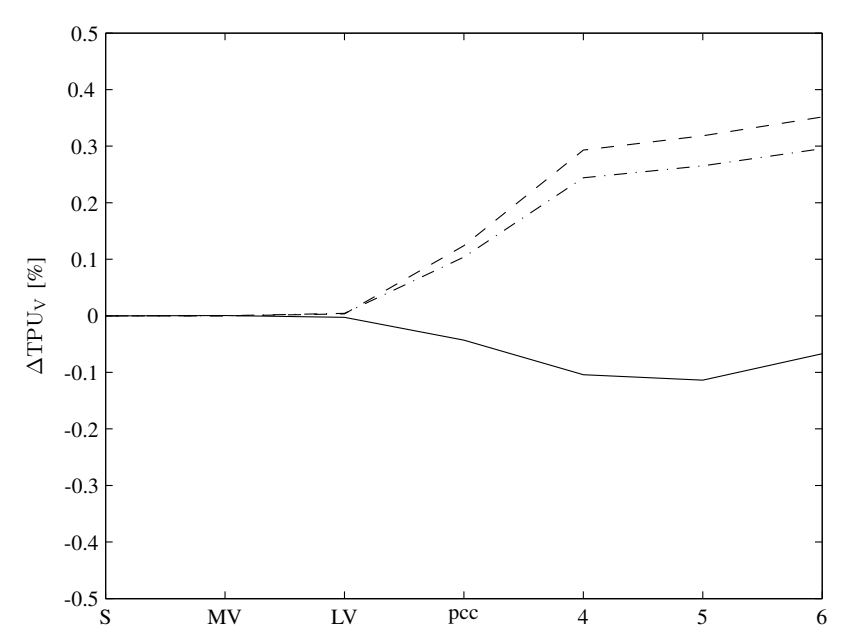

(c) Common sinusoidal inverter

Fig. 4. Influence of the phase of connection on the $\triangle T_{P} U_{V}$ for different types of inverter control 
the differences would be negligible. In Fig. 3 however the $\triangle \mathrm{TPU}_{\mathrm{V}}$ is considered, i.e. also the harmonic voltages are taken into account, which leads to a difference between the control types. The damping inverter has the most beneficial effect, except for the case POC 4, where the common sinusoidal inverter is the best. This good result for the damping inverter finds its origin in the damping of the harmonic voltages.

Remarkable in Fig. 3(c) is the bad result for POC 6, but when Fig. 2(c) is considered, this has an obvious reason, namely, the enlarged harmonic content of the three-phase voltages that has a decisive effect on the $\mathrm{TPU}_{\mathrm{V}}$. At last, one can see an expected increase in unbalance in the case of four DG units connected to phase a.

\section{B. Phase of Connection}

The influence of the phase of connection has been tested by connecting a $3 \mathrm{~kW}$ DG unit to node 6 and this alternated to phase $a, b$ or $c$. The relative change in $\mathrm{TPU}_{\mathrm{V}}$ is shown in Fig. 4. When the DG unit is connected to the most loaded phase, the $\mathrm{TPU}_{\mathrm{V}}$ improves. Furthermore, the same conclusions can be drawn as before, whereas the damping inverter has the best performance.

\section{CONCLUSIONS}

By means of simulations, several inverter control strategies have been compared, considering their influence on the power quality. Two recently formulated indices have been used in order to reveal the influence in more detail. The results show that the damping inverter has a good and predictable effect on the power quality. The common sinusoidal inverter could, in some specific cases, have a better effect on the power quality, but is dependent on the voltage at the POC. Furthermore, the results of the common sinusoidal inverter emphasize the need of taking the phase angles into account when performing harmonic load flow simulations as the diversity and attenuation effect could play an important role in the final result. Moreover, the need for one power quality index is stressed, because if, for the distortion, only the effects in the phase of connection would be considered, this could result in faulty conclusions. For the unbalance, it has been shown that the influence of the harmonics could have an important effect, and therefore, they have to be taken into account. At last, the research presented here shows that the results for the non realistic case of a purely sinusoidal inverter are also predictable, although they are less beneficial than for the damping inverter.

\section{REFERENCES}

[1] D. Menniti, C. Picardi, A. Pinnarelli, and N. Sorrentino, "Reactive power and voltage harmonics compensation of a shunt power active filter," in International symposium on power electronics, electrical drives, automation and motion (SPEEDAM 2006), Taormina, Italy, May 23-26, 2006.

[2] S. Seifossadat, R. Kianinezhad, A. Ghasemi, and M. Monadi, "Quality improvement of shunt active power filter, using optimized tuned harmonic passive filters," in International symposium on power electronics, electrical drives, automation and motion (SPEEDAM 2008), Ischia, Italy, Jun. 11-13, 2008.

[3] T. Hammons, "Integrating renewable energy sources into European grids," Journal of Electrical Power \& Energy Systems ELSEVIER, vol. 30, pp. 462-475, 2008.

[4] L. Degroote, L. Vandevelde, B. Renders, and J. Gyselinck, "Nonlinear transformer model in the frequency domain and with symmetrical components," International Journal for Computation and Mathematics in Electrical and Electronic Engineering (Compel), vol. 27, no. 6, pp. 1418-1437, 2008.

[5] P. Salmerón, R. Herrera, A. Vallés, and J. Prieto, "New distortion and unbalance indices based on power quality analyzer measurements," IEEE Trans. Power Del., vol. 24, no. 2, pp. 501-507, Apr. 2009.

[6] A. von Jouanne and B. Banerjee, "Assessment of voltage unbalance," IEEE Trans. Power Del., vol. 16, no. 4, pp. 782-790, Oct. 2001.

[7] P. Giridhar Kini, R. Bansal, and R. Aithal, "A novel approach toward interpretation and application of voltage unbalance factor," IEEE Trans. Ind. Electron., vol. 54, no. 4, pp. 2315-2322, Aug. 2007.

[8] Y.-J. Wang, "Analysis of effects of three-phase voltage unbalance on induction motors with emphasis on the angle of the complex voltage unbalance factor," IEEE Trans. Energy Convers., vol. 16, no. 3, pp. 270-275, Sep. 2001.

[9] T. Zheng, E. Makram, and A. Girgis, "Evaluating power system unbalance in the presence of harmonic distortion," IEEE Trans. Power Del., vol. 18, no. 2, pp. 393-397, Apr. 2003.

[10] G. Chicco, P. Postolache, and C. Toader, "Analysis of three-phase systems with neutral under distorted and unbalanced conditions in the symmetrical component-based framework," IEEE Trans. Power Del., vol. 22, no. 1, pp. 674-683, Jan. 2007.

[11] G. Chicco, M. Chindris, P. Postolache, and C. Toader, "Analysis of the Steinmetz compensation circuit with distorted waveforms through symmetrical component-based indicators," in 2009 IEEE Bucharest PowerTech Conference, Bucharest, Romania, Jun. 28 -Jul. 3, 2009.

[12] C. Fortescue, "Method of symmetrical coordinates applied to the solution of polyphase networks," Trans. AIEE, vol. 37, no. 2, pp. 1027-1140, Jun. 1918.

[13] W. R. Ryckaert, K. De Gussemé, D. M. Van de Sype, L. Vandevelde, and J. A. Melkebeek, "Damping potential of single-phase bidirectional rectifiers with resistive harmonic behaviour," IEE Proc. Electr. Power Appl., vol. 153, no. 1, pp. 68-74, Jan. 2006.

[14] B. Renders, K. De Gussemé, W. R. Ryckaert, and L. Vandevelde, "Input impedance of grid-connected converters with programmable harmonic resistance," IET Electr. Power Appl., vol. 1, no. 3, pp. 355-361, May 2007.

[15] S. Cobben, "Power quality, implications at the point of connection," Ph.D. dissertation, Technische Universiteit Eindhoven, 2007.

[16] S.-J. Jeon, "Non-sinusoidal power theory in a power system having transmission lines with frequency-dependent resistances," IET Gener. Transm. Distrib., vol. 1, no. 2, pp. 331-339, Mar. 2007.

[17] A. Zobaa, "The optimal passive filters to minimize voltage harmonic distortion at a load bus," IEEE Trans. Power Del., vol. 20, no. 2, pp. 1592-1597, Apr. 2005

[18] F. Gorgette, J. Lachaume, and W. Grady, "Statistical summation of the harmonic currents produced by a large number of single phase variable speed air conditioners: A study of three specific designs," IEEE Trans. Power Del., vol. 19, no. 4, pp. 953-959, Oct. 2000.

[19] A. Mansoor, W. Grady, A. Chowdhury, and M. Samotyj, "An investigation of harmonic attenuation and diversity among single-phase power electronic loads," IEEE Trans. Power Del., vol. 10, no. 1, pp. 467-473, Jan. 1995. 